EPJ Web of Conferences 32, 03007 (2012)

DOI: $10.1051 /$ epjconf/20123203007

(C) Owned by the authors, published by EDP Sciences, 2012

\title{
Recent results and prospects for correlation ECE measurements on TCV
}

\author{
L. Porte ${ }^{1}$, S. Coda ${ }^{1}$, T.P. Goodman ${ }^{1}$, A. Pochelon ${ }^{1}$, V.S. Udintsev ${ }^{2}$, V. Vuille ${ }^{1}$ \\ ${ }^{1}$ Ecole Polytechnique Fédérale de Lausanne (EPFL), Centre de Recherches en Physique des Plasmas, \\ Association Euratom-Confédération Suisse, CH-1015 Lausanne, Switzerland \\ ${ }^{2}$ ITER Organization, Building 519/122, CHD, Diagnostics Division Route de Vinon sur Verdon - \\ 13115 St Paul Lez Durance - France
}

\begin{abstract}
The Tokamak a Configuration Variable (TCV) has a two channel correlation electron cyclotron emission (CECE) radiometer with a line of sight perpendicular to the magnetic field. The antenna pattern of this radiometer limits resolution to $\mathrm{k}_{\theta}<112 \mathrm{~m}^{-1}$ or $\mathrm{k}_{\theta} \rho_{\mathrm{s}}<0.3$ at $500 \mathrm{kHz}$. It can access the region with minor radius $\rho_{\mathrm{vol}}<0.7$. A series of measurements has been made of the turbulence spectra at positive and negative triangularity and as a function of collisionality. Also, a series of measurements has been made as a function of poloidal angle, by varying the plasma vertical position with respect to the antenna, the measurements being made on the same flux surface. It is planned to extensively upgrade the diagnostic by integrating four more channels and acquiring a new front-end for the radiometer. This upgrade should reduce the required number of shots for a radial scan by a factor four and improve the signal-to-noise ratio. It is also planned to use a high gain antenna that will extend access to $\mathrm{k}_{\theta}<174 \mathrm{~m}^{-1}$ or $\mathrm{k}_{\theta} \rho_{\mathrm{s}}<0.5$ at $500 \mathrm{kHz}$. The present system, measurements and the upgrade are described in this paper.
\end{abstract}

\section{Introduction}

Cross magnetic field transport of energy and particles in tokamak plasmas is larger than expected from Coulomb collisions. It is believed that this is due to the presence of micro-instabilities and turbulence [1]. Diagnostic techniques capable of making measurements of core turbulence are thus necessary for the advancement of magnetic fusion. Correlation ECE (CECE) [2] measurements can elucidate the statistical properties of core electron temperature fluctuations in hot dense plasma and CECE is being increasingly used in magnetic fusion experiments. In particular CECE measurements yield the statistical properties of the poloidal component of the turbulent wave vector $\left(\mathrm{k}_{\theta}\right)$ and its radial correlation length. If the CECE radiometer is absolutely calibrated then an absolute measurement of the amplitude of the electron temperature fluctuations can be obtained.

This paper presents, briefly, the TCV tokamak and electron energy transport properties in shaped plasma in TCV. The CECE diagnostic on TCV is then described. Following that a series of CECE

This is an Open Access article distributed under the terms of the Creative Commons Attribution License 2.0, which permits unrestricted use, distribution, and reproduction in any medium, provided the original work is properly cited. 
measurements, made on TCV, is shown and their relation to observed transport properties described. Finally, the TCV CECE radiometer is to receive a major upgrade and the final section of this paper outlines this upgrade.

\section{TCV, electron transport and correlation ECE on TCV}

\subsection{TCV and electron transport}

The tokamak à configuration variable (TCV) was conceived as a tool to study transport of particles and energy, as well as plasma stability, in a wide range of plasma shapes. TCV has sixteen independent plasma shaping coils and, using this extensive shape control, plasma has been created and studied in a multitude of forms, spanning triangularity $(\delta)$ in the range $-0.7<\delta<1.0$ and elongation ( $\kappa)$ in the range $1<\kappa<2.8$

TCV operates with toroidal field less than $1.45 \mathrm{~T}$ and plasma current less than 1MA. The major radius of TCV is $0.8 \mathrm{~m}$ while the minor radius is $0.25 \mathrm{~m}$.

Use has been made of the shaping control to conduct experiments aimed at study of the effect of plasma triangularity, at varying collisionalities, on plasma confinement. Camenen et al [3] described the reduction of thermal diffusivity $\left(\chi_{e}\right)$ both by going from $+v e$ to -ve $\delta$ and by increasing $v_{\text {eff }}\left(\propto n_{e}\right.$ ; for fixed $T_{\mathrm{e}}$ and $Z_{\text {eff }}$ ). As shown in Figure 1, we see the clear reduction in heat diffusivity, by a factor of $\approx 2$, on going from $\delta=+0.4$ to $\delta=-0.4$, at normalised minor radius of 0.55 . The inverse scaling with $v_{\text {eff }}$ is also clearly seen in this figure.

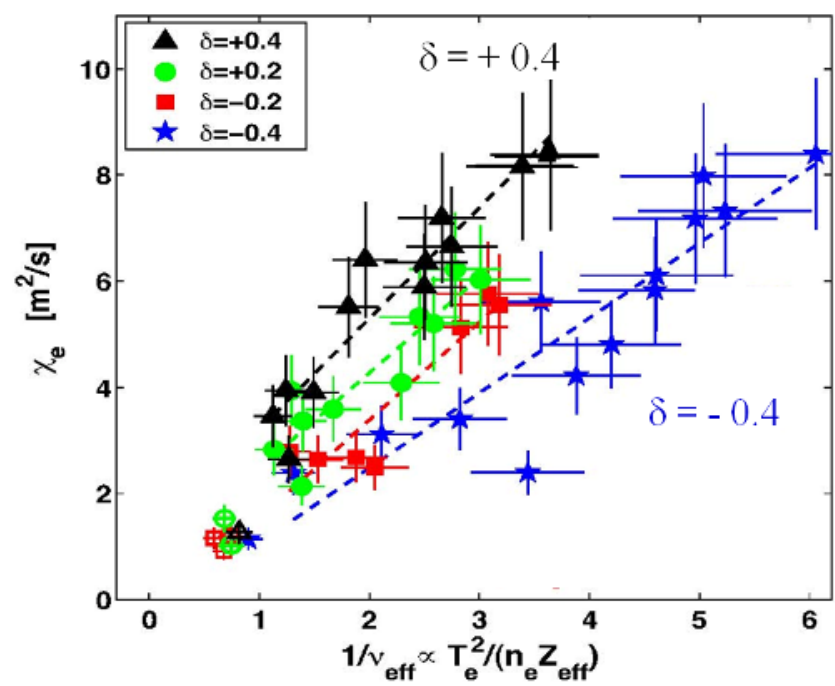

Figure 1: thermal diffusivity shows a clear dependence on plasma shape (triangularity) and the effective collisionality. Confinement improves on going from +ve to -ve triangularity and with increasing density. . (Reprinted with permission from Y. Camenen et al, Nucl. Fus. 47 (2007) 510.)

\subsection{Correlation ECE (CECE) on TCV}

A two channel CECE radiometer has been installed on TCV. This radiometer has a line of sight perpendicular to the magnetic field. The radiometer is coupled to an antenna having an antenna pattern that limits resolution to $\mathrm{k}_{\theta}<112 \mathrm{~m}^{-1}$ or $\mathrm{k}_{\theta} \rho_{\mathrm{s}}<0.3$ at $500 \mathrm{kHz}$.

Radiation is transported from the plasma along $\approx 10 \mathrm{~m}$ of oversized circular waveguide and then delivered to a mixer which covers the frequency range $65 \mathrm{GHz}$ to $81 \mathrm{GHz}$, covering the low field side 
of the machine. The local oscillator frequency is fixed at $63 \mathrm{GHz}$. The mixer output is amplified in low noise intermediate frequency amplifiers $(2-18 \mathrm{GHz})$ and then the power is evenly divided two ways. $50 \%$ of the power is transported to a standard radiometer IF section for profile analysis and the complement is utilized in the CECE system.

The CECE section comprises two narrow, band pass $(170 \mathrm{MHz})$ YIG filters that can be tuned electronically and remotely. The filters' tuning range is from $6 \mathrm{GHz}$ to $18 \mathrm{GHz}$ giving access to normalized minor radii $0<\rho(\psi)<0.7$. After rectification, in Schottky barrier diodes, the signal voltage is amplified and low pass filtered with cut-off frequency $500 \mathrm{MHz}$. Data is acquired at $1 \mathrm{MHz}$ sampling rate.

The CECE radiometer on TCV works using the spectral de-correlation technique [2]. By increasing the spectral distance of two channels, by virtue of the $1 / \mathrm{R}$ dependence of the magnetic field in the plasma, the spatial separation of the two channels increases. In this way the channels sample very spatially close but distinct plasma volumes. The photon noise is immediately decorrelated leaving only the plasma turbulence correlated. Making measurements of the correlation coefficient of the plasma turbulence at various spatial separations yields an estimate of its radial correlation length. The current CECE radiometer, having only two channels, makes radial scans of channel separation rather time consuming.

\section{Recent measurements of correlation ECE made on TCV}

\subsection{Density and Triangularity}

Motivated by the clear dependence of thermal diffusivity on shape and density (for constant temperature), a set of experiments was performed to measure the variation of the turbulence spectra with these plasma quantities. Discharges were established in positive and negative triangularity using ECRH to ensure profile consistency: more EC power was required in the positive delta case to maintain the same electron temperature. For this set of experiments $\mathrm{n}_{\mathrm{e}, \text { local }} \approx 10^{19} \mathrm{~m}^{-3}$ and $\mathrm{T}_{\mathrm{e}, \text { local }} \approx$ $2 \mathrm{keV}$. The CECE frequencies were adjusted so the measurements were made in the gradient region of the profiles where TEM modes were expected to dominate.

Having established a discharge with $\delta=+0.45$ the density was varied from $0.9 \times 10^{19} \mathrm{~m}^{-3}$ to $1.1 \times 10^{19} \mathrm{~m}^{-3}$ : a $20 \%$ increase. This $20 \%$ increase in density caused a dramatic reduction in the cross power spectral density of the turbulence as measured by two channels separated by $0.3 \mathrm{~cm}$ while also causing the radial correlation length of the turbulence to fall from $2.7 \mathrm{~cm}$ to $1.1 \mathrm{~cm}$
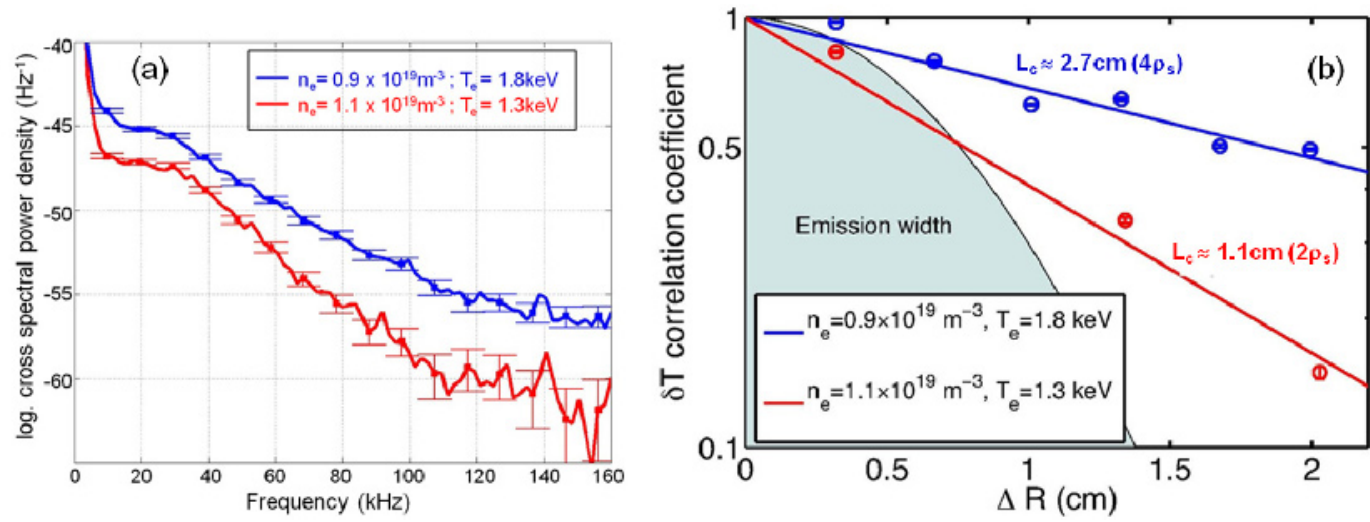

Figure 2: (a) cross power spectral density of $T_{\mathrm{e}}$ fluctuations. By increasing the electron density from 0.9 to 1.1 (tokamak units) a reduction in the CPSD is observed in the frequency range $20 \mathrm{kHz}$ to $>150 \mathrm{kHz}$. (b) at the same time the radial correlation length of the fluctuations is reduced from $2,7 \mathrm{~cm}$ to $1.1 \mathrm{~cm}$ 
Using ECRH to maintain the kinetic profiles, discharges were established at negative triangularity $(\delta=-0.45)$. Again the turbulence spectra were measured and it was seen that in the -ve $\delta$ case the cross power spectra or, equivalently in this case, the electron temperature fluctuations amplitude collapsed. Figure 3 illustrates this. Such was the reduction in the amplitude of the turbulence that it was impossible to extract estimates of $\mathrm{L}_{\mathrm{c}}$ at $-\mathrm{ve} \delta$.

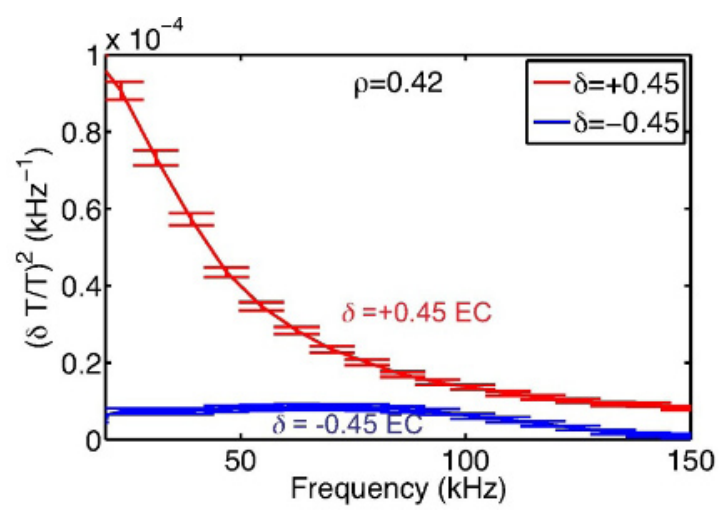

Figure 3: maintaining kinetic profiles using ECRH it was possible to establish discharges at $+v e$ and $-v e \delta$. A very large reduction in cross electron temperature fluctuations was measured on going from $=$ ve to $-v e$ $\delta$.

\subsection{Poloidal Angle}

Bottino [4] produced linear, global calculations of the growth rate of micro-instabilities in TCV plasmas. Spatial variation in the mode structure and amplitude were predicted as a function of poloidal angle on a given flux surface. It was decided to perform a series of measurements of the turbulence spectra and radial correlation length on a fixed flux surface, at different poloidal angles in more or less identical discharges. The aim was simply to see if the CECE diagnostic was capable of measuring differences in the turbulence spectra and in $\mathrm{L}_{c \text {. }}$ as the poloidal angle was changed.

By using a fixed line of sight on the vessel mid-plane and by creating discharges centred at different vertical locations with respect to the vessel mid-plane, it is possible to alter the poloidal angle at which the ECE is sampled. By tuning the CECE frequency it is possible to ensure that the sample volume lies on the same flux surface as the plasma height is changed. Figure 4 illustrates this.

Careful control of the density ensured profile consistency in these discharges. Experiments were conducted with the discharge situated at $\mathrm{Z}_{\text {mag }}=\{0.0,5.0,10.0,17.0\} \mathrm{cm}$. All measurements were made close to $\rho(\psi) \approx 0.7$ where the optical depth was $\approx 3$. Ray tracing calculations verify that the emission came from a volume of plasma very close to this flux surface in all cases. To ensure that the sample volume remained on $\rho(\psi) \approx 0.7$ the CECE frequencies were changed from around $71.5 \mathrm{GHz}$ to $75.5 \mathrm{GHz}$. As seen from the profiles on Figure 4 the sample volume were within the gradient region where TEM turbulence is expected to dominate.

Figure 5(a) summarises the results. It compares measurement made at $Z_{\text {mag }}=0 \mathrm{~cm}$ and $5 \mathrm{~cm}$. A change in the turbulence spectra is observed in moving off axis. There is a rise in fluctuation amplitude in the frequency range $60 \mathrm{kHz}$ to $120 \mathrm{kHz}$ while, at the same time, there is a small increase in the fluctuation amplitude in the low frequency range $20 \mathrm{kHz}$ to $60 \mathrm{kHz}$. A similar change is observed in measurements obtained at $Z_{\text {mag }}=10 \mathrm{~cm}$. The changes in spectral content are substantially larger than the random error in the spectra. Upon reaching $Z_{\text {mag }}=17 \mathrm{~cm}$ electron temperature fluctuation spectra remain broad band $(<120 \mathrm{kHz})$ but are much reduced in amplitude. Figure 5 also shows the radial correlation length deduced from these measurements. 


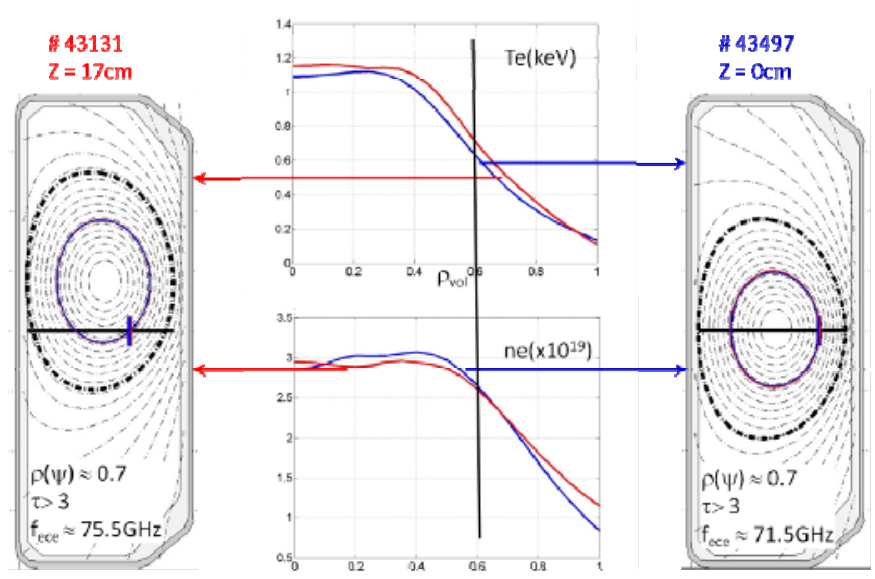

Figure 4: a fixed line of sight centred near the machine centre can probe the turbulence from different regions of the same flux surface (different poloidal angle) by altering vertical position of the plasma and by changing the CECE frequency. In this way a picture can be built up of the spectral properties of the electron temperature fluctuations as a function of poloidal angle and this information may be used to confirm predictions of global models of plasma turbulence. In this example the plasma was shifted from $Z_{\text {mag }}=0$ to $Z_{\text {mag }}=17 \mathrm{~cm}$. At the same time the CECE frequency was changed from $71.5 \mathrm{GHz}$ to $75.5 \mathrm{HGz}$ to ensure that the resonance remained on the same flux surface: $\rho(\psi) \approx 0.7$.
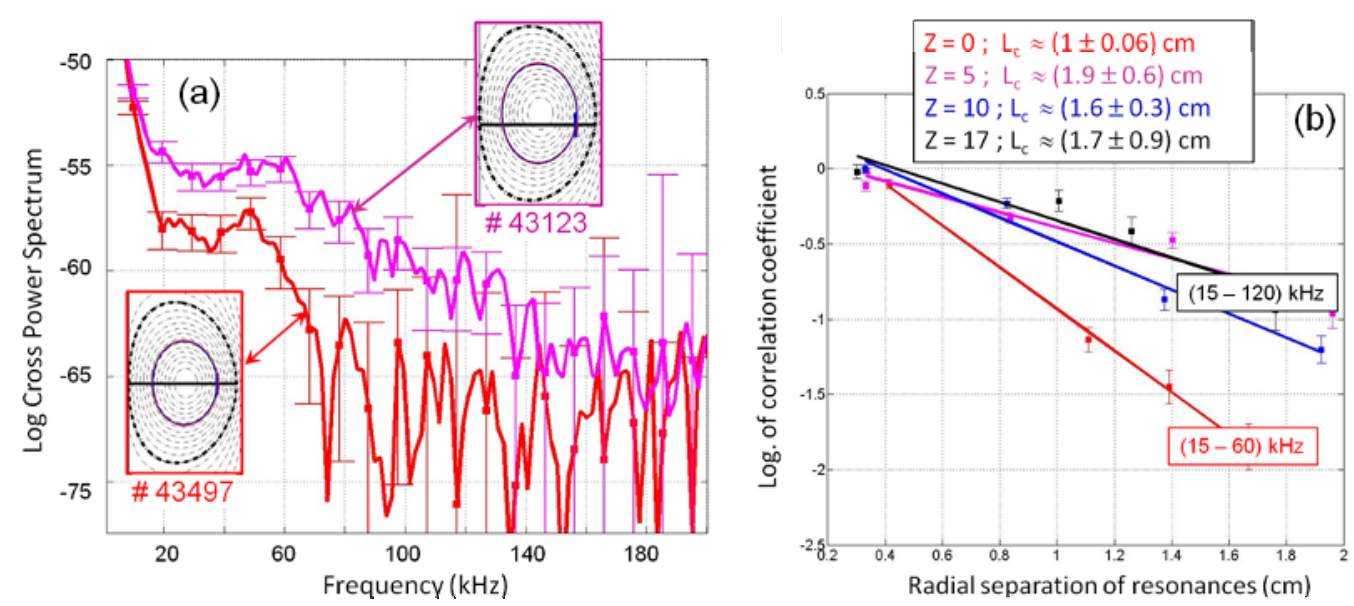

Figure 5: (a) a change in the spectral content of the turbulence is observed upon moving off-axis from $Z_{\text {mag }}=0 \mathrm{~cm}$ to $5 \mathrm{~cm}$. High frequency modes $(60 \mathrm{kHz}<\mathrm{f}<120 \mathrm{kHz})$ appear. The high frequency modes are also present in the measurements made at $Z_{\mathrm{mag}}=10 \mathrm{~cm}$ and $Z_{\mathrm{mag}}=17 \mathrm{~cm}$ with diminishing amplitude. (b) $L_{c}$ for the measurements at all heights. The radial correlation length is substantially shorter at $Z_{\text {mag }}=$ $0 \mathrm{~cm}$ than at $5 \mathrm{~cm}$ or $10 \mathrm{~cm}$. The $L_{c}$ estimation for $Z_{m a g}=17 \mathrm{~cm}$ has a very large uncertainty.

\section{Conclusions from these measurements}

CECE has, so far, proven its worth as a diagnostic of plasma turbulence on TCV. A number of robust measurements have been made.

Clear trends were observed in the effect of collisionality on electron temperature fluctuations: a factor 2 reduction of $\mathrm{L}_{\mathrm{c}}$ for a $20 \%$ increase in density and an overall reduction in the amplitude of the 
electron temperature fluctuations. These observations are coherent with the inverse $v_{\text {eff }}$ scaling of thermal diffusivity.

On going from + ve $\delta$ to - ve $\delta$ the electron temperature fluctuations decreased dramatically and again this is in line with the global trend of confinement improvement while operating at $-\mathrm{ve} \delta$.

The correlation technique has proven itself capable of discerning the turbulent spectra obtained at different poloidal angles on the same flux surface. The correlation lengths obtained from the measurements are useful and indicate, again, that CECE can be used to probe the spatial characteristics of electron temperature fluctuations.

To obtain robust measurements of $\mathrm{L}_{\mathrm{c}}$ using CECE it has been found essential to control the electron density extremely well as $\mathrm{L}_{\mathrm{c}}$ is a strong function of density.

\section{Upgrade for the CECE diagnostic on TCV}

The main drawback for the existing CECE diagnostic has been the fact that it only has two channels. This has made measurements of $\mathrm{L}_{\mathrm{c}}$ long and laborious requiring several 'identical' discharges. At this writing four additional channels are being integrated into the CECE diagnostic. This will allow estimation of $\mathrm{L}_{\mathrm{c}}$ in only one discharge. At the same time the video stage electronics is being upgraded. New higher gain video amplifiers with a true differential output have been designed and built. They will feed the same ADC's as before but using the full differential input. The bandwidth of the filters has been altered slightly to ensure $60 \mathrm{~dB}$ cut-off at $600 \mathrm{kHz}$ ( $3 \mathrm{~dB}$ at close to $450 \mathrm{kHz}$ ) and the data acquisition frequency has been increased to $1.7 \mathrm{MHz}$.

The CRPP is in the process of purchasing two new mixers for the front end of the device so that the CECE diagnostic can be used independently of the standard profile ECE radiometers. This has two advantages: first of all there will be more RF power available to the CECE radiometer (and for the profile radiometers) and secondly the CECE diagnostic can be used on the high resolution steerable antenna already reported by Goodman [5].

Use of the high gain antenna should allow access to $\mathrm{k}_{\theta}<174 \mathrm{~m}^{-1}$ or $\mathrm{k}_{\theta} \rho_{\mathrm{s}}<0.5$ at $500 \mathrm{kHz}$, improving the k-resolution of the system. In addition the ability to steer the antenna in the poloidal plane will open up a whole new vista of potential measurements to be made. The poloidal angle scan will be rendered easier as the discharge will be repeated in the same position in the vessel while moving the antenna. This will ease density control greatly.

In parallel with the material upgrades, a synthetic diagnostic is being put in place. The GENE gyrokinetic code [6], in its local and global incarnations, will be coupled to an interface for the simulation of diagnostic response.

With these substantial upgrades, CECE on TCV will be very well placed to contribute to the TCV programme but also to the larger European transport task groups.

This work was supported in part by the Swiss National Science Foundation.

\section{References}

[1] F.Wagner \& U. Stroth; "Transport in toroidal devices - The experimentalist's view"; Plasma Phys. Control Fusion, 35, 1321(1993)

[2] C.Watts; "ECE Correlation radiometry techniques for detection of core electron temperature fluctuations"; Fusion Sci. Technol., 52, 176 (2007)

[3] Y. Camenen et a; "Impact of plasma triangularity and collisionality on electron transport in TCV L-mode plasmas"; Nucl. Fus. 47 (2007) 510 - 516.

[4] Bottino A 2005 Modelling of global electrostatic microinstabilities in tokamak: effects of $E \times B$ flow and magnetic shear PhD Thesis 2983 Ecole Polytechnique F'ed'erale de Lausanne

[5] T.P. Goodman et al Fusion Sci. Technol. 53, 196 (2008)

[6] T. Görler et al., "The Global Version of the Gyrokinetic Turbulence Code GENE"', J. Comp. Phys. 230, 7053-7071 (2011). 